\title{
Detection of Mechanical Injury and Physiological Breakdown of Cucumbers Using Delayed Light Emission
}

\author{
Judith A. Abbott \\ Instrumentation and Sensing Laboratory, Agricultural Research Service, U.S. Department of \\ Agriculture, Beltsville, MD 20705
}

\author{
A. Raymond Miller \\ Department of Horticulture, Ohio Agricultural Research and Development Center, The Ohio State \\ University, Wooster, OH 44691
}

T. Austin Campbell

Soybean and Alfalfa Research Laboratory, Agricultural Research Service, U.S. Department of Agriculture, Beltsville, MD 20705

\begin{abstract}
Additional index words. Cucumis sativus, postharvest, chilling stress, storage, quality, bruising, electron microscopy, chloroplasts, instrumentation

Abstract. Mechanical stress received by pickling cucumbers (Cucumis sativus L.) during harvest can cause physiological degeneration of the placental tissues, rendering the cucumbers unsuitable for use in some pickled products. Cucumbers were subjected to controlled stresses by dropping and rolling under weights to induce such degeneration. Following storage at various temperatures for 0,24 , and 48 hours, refreshed delayed light emission from chlorophyll (RDLE) was measured and transmission electron micrographs of chloroplasts were made. Mechanical stress rapidly suppressed RDLE and induced accumulation of starch granules within the chloroplasts. Rolling usually had a greater effect on RDLE than did dropping. After 48 hours, RDLE suppression persisted; starch granules were no longer evident in chloroplasts from mechanically stressed fruit, but very electron-dense inclusions had developed in the chloroplasts. Storage temperatures affected RDLE levels but had minimal interaction with stress responses. Cucumber lots subjected to excessive mechanical stress likely could be detected using RDLE measurement.
\end{abstract}

The processing quality of cucumbers is a major concern of the pickling industry. Mechanical injury can cause physiological breakdown during postharvest storage and processing, typified by sloughing of the placental tissue. Such sloughing renders the pickled products unacceptable for marketing.

Decreased cucumber quality as evidenced by tissue softening and deterioration has been linked to mechanical injury (Marshall et al., 1972; Miller et al., 1987). This deterioration involves the induction of cell wall-degrading enzymes (Miller et al., 1987), anodic peroxidase isozymes (Miller and Kelly, 1989), and changes in the sugar composition of cell wall-associated polysaccharides (Miller, 1989). Although research has increased our understanding of the physiological and biochemical responses to stress, a practical method has not been developed to distinguish effectively between mechanically stressed and control cucumbers.

Abbott and Massie (1985) found that cucumber fruit exhibit delayed light emission (DLE). DLE results primarily from relaxation of chlorophyll molecules that have been excited by the back-reaction of photosystem II (Ellenson, 1986; Rutherford et al., 1984; Strehler and Arnold, 1951). DLE thus depends on a contiguous and functional photosynthetic apparatus (Arnold and Davidson, 1954; Ellenson, 1986), and factors that alter or dis-

\footnotetext{
Received for publication 18 Dec. 1989. Supported in part by state and federal funds appropriated to the Ohio Agricultural Research and Development Center, The Ohio State Univ. We thank C. Velbinger, M. Jameson, and B. Borchart for technical assistance; R. Whitmoyer and E. Kretzschmar, Electron Microscope Lab, OARDC, for preparing and photographing TEM samples; and L. Douglas, USDA-ARS Statistical Consulting Lab, for assistance with repeated measures analyses of variance. Mention of proprietary products does not imply endorsement of the product named or criticism of similar products not mentioned. The cost of publishing this paper was defrayed in part by the payment of page charges. Under postal regulations, this paper therefore must be hereby marked advertisement solely to indicate this fact.
}

rupt chloroplast membranes may decrease DLE values. DLE following a single exciting flash has a rapid exponential decay. Refreshing the stimulation of chloroplasts by rapid repetitive excitation and measurement (RDLE) results in time curves such as Fig. 1 (Abbott and Massie, 1985). Abbott and Massie (1985) showed that RDLE measurements could be used for the early detection of chilling injury on fresh-market cucumbers.

Miller et al. (1987) noted that water-soaked lesions were present in the skin of mechanically stressed cucumbers immediately after treatment, indicating membrane damage at the cellular level. Since mechanical stress may also have affected skin chloroplast integrity or function, DLE could have been reduced. Hence, the present study was undertaken to 1) examine the effects of mechanical stress and subsequent storage on RDLE and chloroplast structure, and 2) determine the feasibility of using RDLE for early detection of mechanical damage to and chilling injury of pickling cucumbers.

\section{Materials and Methods}

Cucumber treatment. Cucumbers ('Heinz 3534') were fieldgrown at the Ohio Agricultural Research and Development Center, Wooster. For each experiment, commercial size 3 cucumbers (37 to $55 \mathrm{~mm}$ diameter, 14 to 16 days postanthesis) were carefully hand-harvested and washed with cool tap water. Fruit for RDLE studies were transported at $\approx 23 \mathrm{C}$ in styrofoam boxes to Beltsville, Md. Stress experiments began $\leq 24 \mathrm{hr}$ after harvest.

In each of three experiments, two forms of stress were applied, dropping and rolling, shown by Miller et al. (1987) to

Abbreviations: DLE, delayed light emission; MAX, maximum RDLE; RDLE, refreshed delayed light emission. 
simulate the stress caused by mechanical harvesting. Cucumbers were hand-held horizontally and dropped $1 \mathrm{~m}$ to a tiled concrete floor zero or six times; radial orientation was random. Cucumbers with visible cracks were culled. The remaining cucumbers were placed individually under a $30-\mathrm{cm}$-square board supporting the masses specified in Table 1 and the board was hand-rolled back and forth over the fruit $\approx 30$ cycles in 60 sec. A $0-\mathrm{kg}$ roll weight indicates that fruit were not rolled. Cucumbers were placed in plastic trays loosely encased in polyethylene bags to minimize water loss. RDLE was measured on the same cucumbers after storage durations of 0,24 , and $48 \mathrm{hr}$ at the temperatures indicated in Table 1 . All cucumbers were at $\approx 23 \mathrm{C}$ during treatment and initial measurement, but were near their assigned storage temperatures when measured at 24 and $48 \mathrm{hr}$.

Refreshed delayed light emission measurement. RDLE was measured using a prototype instrument described by Abbott and Massie (1985). Before RDLE measurement, cucumbers were dark-equilibrated for $\geq 1 \mathrm{hr}$ at $\approx 23 \mathrm{C}$. RDLE was measured at the longitudinal midpoint on two dark-green cucumber surfaces. Each 15-sec RDLE measurement comprised one thousand 15ms cycles, 7-ms illumination and 7-ms RDLE detection in the dark with $0.5 \mathrm{~ms}$ between phases. Precision to four significant figures is shown only where essential.

Transmission electron microscopy. Cucumbers harvested at the same age and subjected to selected stress treatments were sampled for electron microscopy at Wooster. Two tissue samples (5-mm cubes) from each of three cucumbers per treatment were removed from the longitudinal midpoint of the two darkgreen surfaces. Fifteen minutes elapsed from initiation of mechanical stress to immersion of tissue samples in fixitive. Samples were fixed in a solution of 3\% glutaraldehyde, $2 \%$ paraformaldehyde, and $1.5 \%$ acrolein in $0.1 \mathrm{M}$ sodium phosphate $(\mathrm{pH} \mathrm{7.3)}$ at $4 \mathrm{C}$. The samples were washed with buffer, post-fixed in $2 \%$ osmium tetroxide in sodium phosphate $(0.1$ $\mathrm{M}, \mathrm{pH}$ 7.3) for $3 \mathrm{hr}$, washed again with buffer, stained overnight in $1 \%$ uranyl acetate, dehydrated in an ethanol-acetone series, then embedded in Spurr plastic and polymerized at 70C (Spurr, 1969). Specimen blocks were sectioned to a thickness of $10 \mathrm{~nm}$ with a Sorvall MT-2B ultramicrotome, retrieved on Formvarcoated 300-mesh grids, and stained with $0.5 \%$ uranyl acetate and $0.1 \%$ lead acetate in water. Specimens were photographed with a Phillips 201 electron microscope at $80 \mathrm{kV}$.

Statistical design. In each of three factorial experiments, treatments (stresses and temperatures) were arranged in a completely randomized design with five replications (Table 1); experimental units were individual cucumbers. Cucumbers were nested within stresses $\times$ temperature; sides, within cucumbers; and durations, within sides.

Statistical analyses. RDLE amplitudes from 10 points along the RDLE time curve $(0.015,0.060,0.135,0.285,1.485,2.985$, $4.485,5.985,7.485$, and 14.985 see, selected based on inspection and previous experiments) and the maximum RDLE (MAX) were analyzed separately by single degree of freedom contrast or orthogonal polynomial analysis of variance (ANOVA), as appropriate (Steel and Torrie, 1960; SAS, 1988). The 10 points along the RDLE curve were collectively analyzed by repeated measures ANOVA (which furnishes an intercorrelation matrix), using time as the repeated variable (SAS, 1988). MAX was similarly analyzed using storage duration as the repeated variable.

\section{Results and Discussion}

RDLE amplitudes increased from the initial value at 0.015 see, reached a maximum between 4.5 and 9 see, and then de-
Table 1. Mechanical stress and storage temperature combinations used to simulate harvest abuse of pickling cucumbers in Expts. 1 through 3. Mechanical stress was applied by dropping cucumbers $1 \mathrm{~m}$ the specified number of times and/or by rolling individual cucumbers under a board supporting the specified mass for $60 \mathrm{sec}$; 0 roll weight indicates that fruit were not rolled.

\begin{tabular}{|c|c|c|c|c|c|c|c|c|c|c|}
\hline \multirow{3}{*}{$\frac{\text { Expt. }^{\mathrm{z}}}{1}$} & \multicolumn{6}{|c|}{ Stresses } & & & & \\
\hline & \multicolumn{2}{|c|}{$\begin{array}{c}\text { Drops } \\
\text { (no.) }\end{array}$} & \multicolumn{4}{|c|}{$\begin{array}{c}\text { Roll wt } \\
\text { (kg) }\end{array}$} & \multicolumn{4}{|c|}{$\begin{array}{l}\text { Temperature } \\
\left({ }^{\circ} \mathrm{C}\right)\end{array}$} \\
\hline & 0 & 6 & 0 & & & 10 & 2.5 & 10 & 25 & 38 \\
\hline 2 & 0 & 6 & 0 & & & 10 & & 10 & 25 & 38 \\
\hline 3 & 0 & 6 & 0 & 3 & 6 & 10 & & & & 38 \\
\hline
\end{tabular}

${ }^{2}$ Only 0 drops $\times 0 \mathrm{~kg}$ and 6 drops $\times 10 \mathrm{~kg}$ were tested in Expt. 1; complete factorial combinations were tested in Expts. 2 and 3.

${ }^{y}$ All cucumbers were at $\approx 23 \mathrm{C}$ for mechanical stress treatments. Initial measurements were made at $\approx 23 \mathrm{C} ; 24$ - and 48 -hr measurements were made after $\approx 1 \mathrm{hr}$ dark-equilibration at $\approx 23 \mathrm{C}$.

creased until measurement ceased at $15 \mathrm{sec}$ (Fig. 1 A-D). Increasing RDLE amplitudes up to the maximum are theoretically due to more electrons flowing back into photosystem II chlorophyll from distant but contiguous electron pools (e.g., plastoquinone, plastocyanin, cytochromes, ferredoxin) (Ellenson, 1986; Strehler and Arnold, 1951), and are presumably caused by incomplete energy dissipation between the repeated light exposures. The small shoulders may reflect filling of these distant electron pools. The decline of RDLE amplitudes from the maximum to the termination of measurement could be due to bleaching of chlorophyll (R. Hangarter, personal communication).

Not surprisingly, repeated measures ANOVA over the 10 selected times within measurements indicated that times were interrelated (tests for sphericity applied to orthogonal components significant at $P=0.0001$ for Expts. 1 through 3). However, examination of the partial correlation matrixes indicate that early times ( 0.015 through 0.285 see) were not closely correlated to subsequent times (1.485 through 15 see; data not shown). Amplitudes at early times were relatively low, so absolute differences among stress treatments were small, although significant. While actual amplitudes differed among times from 1.485 to 15 see, amplitudes at all times in that range responded significantly and similarly to treatments. Maximum amplitudes are representative of the 1.485 to 15 -sec portion of the curve; therefore, only MAX responses are presented.

MAX usually increased very slightly over the $48 \mathrm{hr}$ of testing. However, measuring the progression of stress response over time was not our primary interest. Cucumbers would probably be measured at only one time, either upon delivery to the packer or just before processing. Therefore, we examined each storage duration separately to determine whether mechanical injury was detectable at that time. Analyses indicated that it was not necessary to compensate for repeatedly measuring the same sites at 24-hr intervals (tests for sphericity applied to orthogonal components in repeated measures ANOVA were $P=0.09,0.30$, and 0.31 for Expts. 1 through 3, respectively).

We anticipated that stress responses would be enhanced by high storage temperatures. This was not the case, although storage temperatures exerted some significant effects on RDLE (Tables 2 through 4, Fig. 1 A-D). In Expt. 1, regardless of mechanical stress treatment, RDLE curves from fruit stored at a chilling temperature $(2.5 \mathrm{C})$ had a strong early shoulder and lower amplitudes at subsequent times (Fig. 1A) than did curves from comparable cucumbers stored at nonchilling temperatures 

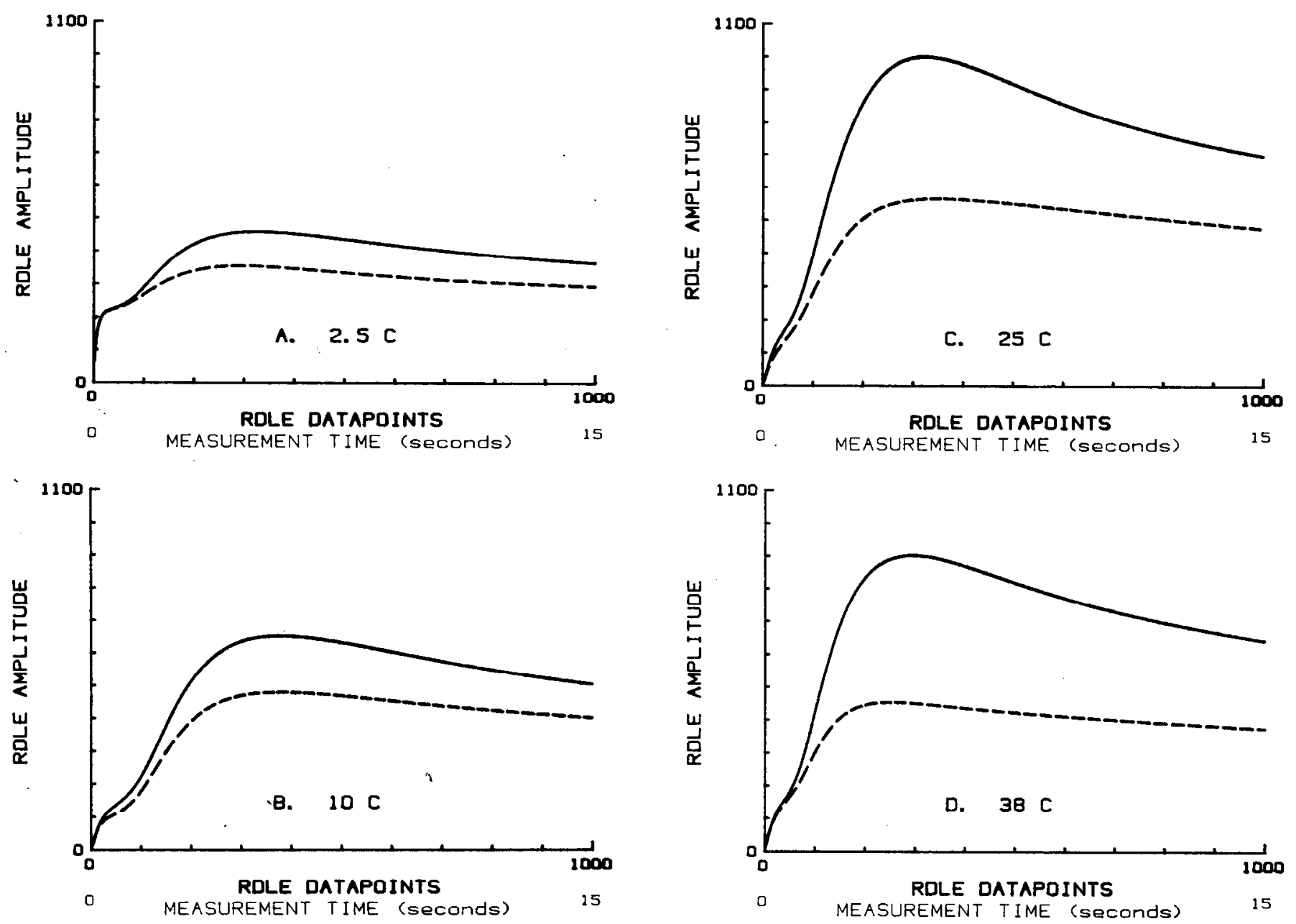

Fig. 1. RDLE curves for cucumbers subjected to controlled mechanical stresses (six drops and 10-kg roll weight) (-----) or to no mechanical stress (-) and held for $48 \mathrm{hr}$ at the temperatures indicated. Each curve is the mean of 10 measurements (five cucumbers $\times$ two green sides) from Expt. 1.

Table 2. Significance (probabilities, $1 \mathrm{df}$ ) of mechanical stress and temperature effects on MAX of cucumbers in Expt. 1.

\begin{tabular}{|c|c|c|c|c|c|c|c|c|}
\hline \multirow{3}{*}{$\begin{array}{l}\text { Source } \\
\text { of } \\
\text { variation } \\
\end{array}$} & \multicolumn{4}{|c|}{ Including $2.5 \mathrm{C}$} & \multicolumn{4}{|c|}{ Excluding $2.5 \mathrm{C}$} \\
\hline & \multirow[b]{2}{*}{$\mathrm{df}$} & \multicolumn{3}{|c|}{ Duration (hr) } & \multirow[b]{2}{*}{$\mathrm{df}$} & \multicolumn{3}{|c|}{ Duration (hr) } \\
\hline & & 0 & 24 & 48 & & 0 & 24 & 48 \\
\hline Stresses (S) & 1 & 0.0001 & 0.0008 & 0.0001 & 1 & 0.0002 & 0.0020 & 0.0001 \\
\hline Temperature $(\mathrm{T})$ & 1 & 0.0542 & 0.1533 & 0.0001 & 1 & 0.0248 & 0.1906 & 0.0934 \\
\hline $\mathrm{T}^{2}$ & 1 & 0.9770 & 0.6255 & 0.0036 & 1 & 0.0815 & 0.2013 & 0.0320 \\
\hline$T^{3}$ & 1 & 0.0207 & 0.2180 & 0.5083 & & & & \\
\hline $\mathrm{S} \times \mathrm{T}$ & 1 & 0.3203 & 0.6847 & 0.0114 & 1 & 0.5577 & 0.8587 & 0.1044 \\
\hline $\mathrm{S} \times \mathrm{T}^{2}$ & 1 & 0.4238 & 0.3572 & 0.4748 & 1 & 0.2978 & 0.2010 & 0.3843 \\
\hline $\mathrm{S} \times \mathrm{T}^{3}$ & 1 & 0.4435 & 0.3686 & 0.5191 & & & & \\
\hline
\end{tabular}

${ }^{z}$ See Table 1 for treatments. Note that Expt. 1 contained only roll weight/drop combinations $0 / 0$ and $10 / 6$.

${ }^{y}$ Superscripts $\left({ }^{2,3}\right)$ refer to quadratic and cubic effects.

(Fig. 1 B-D), similar to results obtained by Abbott and Massie (1985) with chilling exposure of fresh-market cucumbers. Analyses for Expt. 1 are shown with and without the chilling treatment (Table 2). Chilling was omitted from subsequent experiments. Excluding chilling injury, effects and interactions of temperature (Tables 2 and 3) were small and too erratic to be interpretable. Therefore, temperature effects were omitted in Expt. 3.
RDLE curves were lower for mechanically stressed fruit than for nonstressed fruit at durations 0 and $24 \mathrm{hr}$ in all experiments (Tables 2, 3, and 5; Fig. 1 A-D). RDLE curves remained significantly lower for stressed cucumbers after $48 \mathrm{hr}$ in Expts. 1 and 3, and the means indicate a similar trend in Expt. 2 as well. Roll weight usually had a greater effect on RDLE than did dropping, probably because the injury inflicted by rolling was more uniformly distributed, thereby increasing the probability 
Table 3. Significance (probabilities, $1 \mathrm{df}$ ) of mechanical stress and temperature effects on MAX of cucumbers in Expt. 2.

\begin{tabular}{lcccc}
\hline \hline \multirow{2}{*}{$\begin{array}{l}\text { Source of } \\
\text { variation }\end{array}$} & & \multicolumn{3}{c}{ Duration (hr) } \\
\cline { 4 - 5 } & df & 0 & 24 & 48 \\
\hline Rolled (R) & 1 & 0.0001 & 0.0015 & 0.1513 \\
Dropped (D) & 1 & 0.0001 & 0.0665 & 0.1214 \\
$\mathrm{R} \times \mathrm{D}$ & 1 & 0.0965 & 0.7400 & 0.8788 \\
$\mathrm{~T}$ & 1 & 0.6396 & 0.0102 & 0.1721 \\
$\mathrm{~T}^{2}$ & 1 & 0.1532 & 0.0570 & 0.0922 \\
$\mathrm{~T} \times \mathrm{R}$ & 1 & 0.2672 & 0.4287 & 0.7740 \\
$\mathrm{~T}^{2} \times \mathrm{R}$ & 1 & 0.7175 & 0.2475 & 0.0634 \\
$\mathrm{~T} \times \mathrm{D}$ & 1 & 0.2048 & 0.0145 & 0.1687 \\
$\mathrm{~T}^{2} \times \mathrm{D}$ & 1 & 0.7052 & 0.3725 & 0.9089 \\
$\mathrm{~T} \times \mathrm{R} \times \mathrm{D}$ & 1 & 0.1223 & 0.0200 & 0.5433 \\
$\mathrm{~T}^{2} \times \mathrm{R} \times \mathrm{D}$ & 1 & 0.7103 & 0.1810 & 0.6302 \\
\hline
\end{tabular}

${ }^{2}$ See Table 1 for treatments. Superscript $\left({ }^{2}\right)$ refers to quadratic effects.

Table 4. Significance (probabilities, $1 \mathrm{df}$ ) of mechanical stress and temperature effects on MAX of cucumbers in Expt. 3.

\begin{tabular}{|c|c|c|c|c|}
\hline \multirow{2}{*}{$\begin{array}{l}\text { Source of } \\
\text { variation }^{z}\end{array}$} & \multirow[b]{2}{*}{$\mathrm{df}$} & \multicolumn{3}{|c|}{ Durations (hr) } \\
\hline & & 0 & 24 & 48 \\
\hline Roll wt & 1 & 0.0001 & 0.0465 & 0.0072 \\
\hline Roll wt ${ }^{2}$ & 1 & 0.5197 & 0.4863 & 0.4549 \\
\hline Roll wt ${ }^{3}$ & 1 & 0.9191 & 0.7530 & 0.5237 \\
\hline Dropped (D) & 1 & 0.5145 & 0.9968 & 0.5069 \\
\hline Roll wt $\times$ D & 1 & 0.5314 & 0.9806 & 0.4226 \\
\hline Roll $w t^{2} \times D$ & 1 & 0.4849 & 0.1597 & 0.6480 \\
\hline Roll $w t^{3} \times D$ & 1 & 0.4652 & 0.7494 & 0.6073 \\
\hline
\end{tabular}

${ }^{2}$ See Table 1 for treatments. Superscripts $\left({ }^{2},{ }^{3}\right)$ refer to quadratic and cubic effects.

Table 5. Mean refreshed delayed light emission maxima (MAX $\times$ $10^{-2}$, arbitrary units) and coefficients of variation (cv) for mechanically stressed cucumbers stored for 0,24 , and $48 \mathrm{hr}^{2}$.

\begin{tabular}{|c|c|c|c|c|c|c|c|c|c|}
\hline \multirow{3}{*}{$\begin{array}{l}\text { Meehanical } \\
\text { stress }\end{array}$} & \multicolumn{3}{|c|}{ Expt. 1} & \multicolumn{3}{|c|}{ Expt. 2} & \multicolumn{3}{|c|}{ Expt. 3} \\
\hline & \multicolumn{9}{|c|}{ Storage duration (hr) } \\
\hline & 0 & 24 & 48 & 0 & 24 & 48 & 0 & 24 & 48 \\
\hline \multicolumn{10}{|l|}{ Roll wt (kg) } \\
\hline 0 & 71 & 77 & 76 & 85 & 86 & 81 & 112 & 124 & 144 \\
\hline 3 & & & & & & & 105 & 126 & 13 \\
\hline 6 & & & & & & & 97 & 115 & 11 \\
\hline 10 & 52 & 58 & 48 & 65 & 73 & 73 & 81 & 101 & 110 \\
\hline \multicolumn{10}{|l|}{ Drops (no.) } \\
\hline 0 & 71 & 77 & 76 & 83 & 83 & 81 & 101 & 117 & 128 \\
\hline 6 & 52 & 58 & 48 & 67 & 75 & 73 & 97 & 117 & 121 \\
\hline $\mathrm{CV}$ & 20 & 25 & 26 & 18 & 19 & 26 & 23 & 34 & 35 \\
\hline
\end{tabular}

${ }^{2}$ See Table 1 for treatments and Tables 2-4 for analyses of variance. Because data are averaged over storage temperatures in each experiment, including 2.5C in Expt. 1, comparisons should be made only within experiments.

of bruising at the RDLE measurement site. When several roll weights were tested in Expt. 3, the linear component was significant (Table 4, Fig. 2), but the quadratic and cubic components were not. Note that a response to dropping was apparent in Fig. 2 only at $\mathrm{O}-\mathrm{kg}$ roll weight. In cucumbers that actually were rolled, the response to the additional abuse of dropping was trivial. Growing conditions apparently affected susceptibility to mechanical injury; more fruit were culled due to cracking after dropping in Expt. 2 than in the other experiments (data not shown).

Lower RDLE amplitudes result from a reduction in the num-

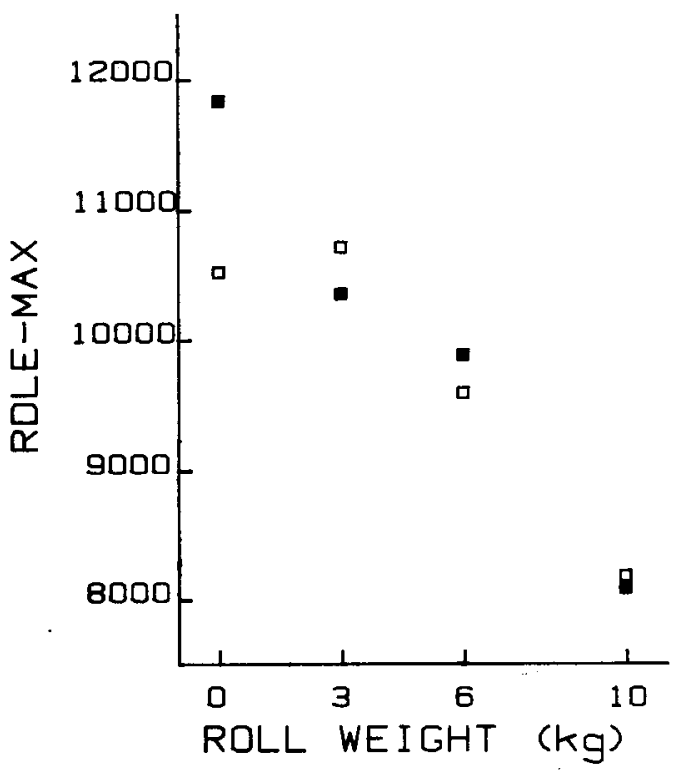

Fig. 2. Mechanical stress effects on $M A X=1 \mathrm{hr}$ after stress treatment (Expt. 3). Differences between zero ( $\square$ ) and six ( $\square$ ) drops were nonsignificant at each roll weight except at $0 \mathrm{~kg}$. Each point is the mean of 10 measurements (five cucumbers $\times$ two green sides).

ber of electrons flowing back into photosystem II. This reduction could be accounted for by several mechanisms, including a disruption of the photosystem II light-harvesting capabilities or the uncoupling of photosystem II from subsequent electron acceptors. The latter hypothesis is supported by the work of Keck and Boyer (1974), who showed that photosynthetic electron transport decreased during water stress. Since these mechanisms could result from structural changes within the chloroplasts caused by bruising, we examined cucumber skin chloroplasts using transmission electron microscopy.

Transmission electron micrographs of chloroplasts from control and mechanically stressed (6 drops $\times 10-\mathrm{kg}$ roll weight) cucumbers after 0 and $48 \mathrm{hr}$ of storage show that chloroplasts from control cucumbers appeared normal, with well-defined granal stacks connected by stromal lamellae, regardless of storage time (Fig. $3 \mathrm{~A}$ and C). Immediately after treatment, chloroplasts from mechanically stressed cucumbers also appeared normal except for an accumulation of starch granules within the stroma (Fig. 3B). The rapid appearance of starch indicates a change in chloroplast functioning and suggests decreased availability of orthophosphate, possibly due to increased photophosphorylation (noncyclic and/or cyclic) or decreased orthophosphate translocation into the chloroplasts (Preiss, 1982). The relation of starch accumulation to decreased RDLE amplitudes is unclear, but may be a result of feedback inhibition of electron transport through the chloroplast membrane. This hypothesis warrants further investigation.

Forty-eight hours after treatment, starch granules were no longer evident in chloroplasts from mechanically stressed fruit, but other structural changes were observed (Fig. 3D). Most striking was the appearance of very electron-dense inclusions within the chloroplasts. Although the chemical composition of these inclusions is not known, they resemble structures identified as plastoglobuli by Vapaavuori et al. (1984). These investigators reported the appearance of large plastoglobuli in willow leaf chloroplasts in response to severe water stress. The presence of plastoglobuli in cucumber skin chloroplasts following mechanical stress suggests that their appearance may be a general response of photosynthetic tissues to 


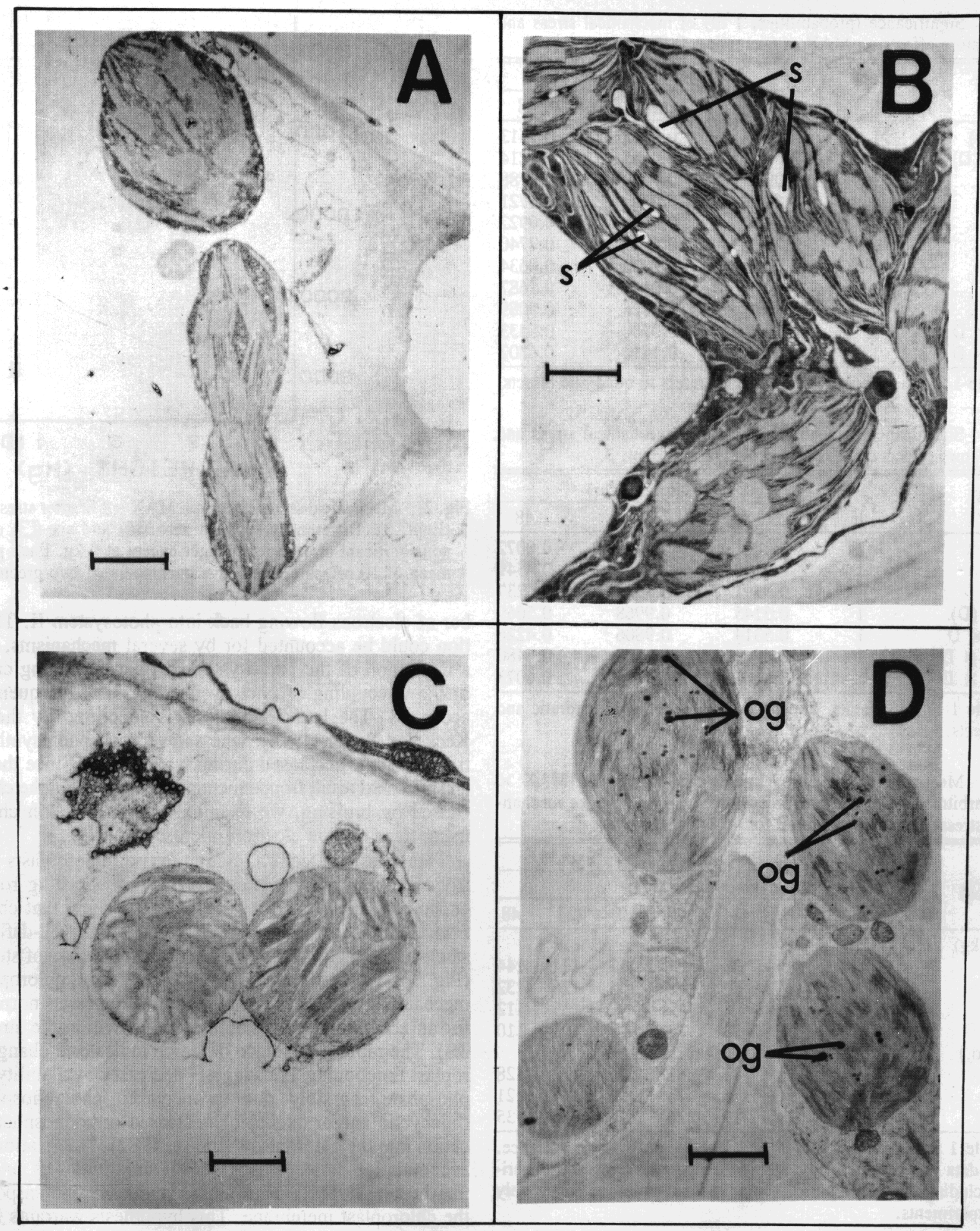

Fig. 3. Transmission electron micrographs $(8500 \times)$ of chloroplasts from nonstressed and mechanically stressed (six drops $\times 10-\mathrm{kg}$ roll weight) cucumbers after 0 and 48 hr. (A) Nonstressed, 0 hr. (B) Stressed, 0 hr. (C) Nonstressed, 48 hr. (D) Stressed, 48 hr. Horizontal bar represents $1 \mu \mathrm{m}, \mathrm{s}=$ starch, og = plastoglobuli.

several types of stress. Further, the granal stacks in these chloroplasts had degenerated, resulting in a diffuse thylakoid membrane system. This observation indicates a loss of organization of the light-harvesting system.

Simulated harvest abuse of pickling cucumbers reduced RDLE from skin chloroplasts. Mechanically stressed cucumbers could be discriminated from nonstressed cucumbers using RDLE am- plitude at the maximum or at any specific measurement time later than $1.485 \mathrm{sec}$ up to termination at $15 \mathrm{sec}$. At the levels of mechanical stress we tested, injury could be detected soon after $(\approx 1 \mathrm{hr})$ and for at least 1 day after stress treatments. The value and variablity of pickling cucumbers and the time requirements for dark equilibration and RDLE measurement make sorting of individual pickling cucumbers impractical with pres- 
ent RDLE equipment. However, RDLE could be used as an inspection tool by processors to identify loads of cucumbers that had been subjected to sufficient stress to induce physiological breakdown. These cucumbers could then be diverted to appropriate uses.

\section{Literature Cited}

Abbott, J.A. and D.R. Massie. 1985. Delayed light emission for early detection of chilling in cucumber and bell pepper fruit. J. Amer. Soc. Hort. Sci. 110:42-47.

Arnold, W.A. and J.B. Davidson. 1954. The identity of the fluorescent and delayed light emission spectra in Chlorella. J. Gen. Physiol. 37:677-684.

Ellenson, J.L. 1986. Flash-induced delayed light emission patterns of red kidney bean leaves: evidence of a new component dependent upon tissue integrity. Plant Physiol. 80:988-991.

Keck, R.W. and J.S. Boyer. 1974. Chloroplast response to low leaf water potentials. 111. Differing inhibition of electron transport and photophosphorylation. Plant Physiol. 53:474479.

Marshall, D. E., B.F. Cargill, and J.H. Levin. 1972. Physical and quality factors of pickling cucumbers as affected by mechanical harvesting. Trans. Amer. Soc. Agr. Eng. 15:604-608, 612.

Miller, A.R. 1989. Mechanical stress-induced changes in the sugar composition of cell walls from cucumber fruit tissues. Photochemistry 28:389-392.
Miller, A.R. and T.J. Kelley. 1989. Mechanical stress stimulates peroxidase activity in processing cucumber fruit. HortScience 24:650652.

Miller, A. R., J.P. Dalmasso, and D.W. Kretchman. 1987. Mechanical stress, storage time, and temperature influence cell wall-degrading enzymes, firmness, and ethylene production by cucumbers. J. Amer. Soc. Hort. Sci. 112:666-671.

Preiss, J. 1982. Regulation of the biosynthesis and degradation of starch. Annu. Rev. Plant Physiol. 33:431-454.

Rutherford, A. W., Govindjee, and Y. Inoue. 1984. Charge accumulation and photochemistry in leaves studied by thermoluminescence and delayed light emission. Proc. Natl. Acad. Sci. USA 81:11071111.

SAS Institute, Inc. 1988. SAS/STAT user's guide. Release 6.03. (ed.). SAS Institute, Inc., Cary, N.C.

Spurr, A.R. 1969. A low viscosity epoxy resin embedding medium for electron microscopy. J. Ultrastructure Res. 26:31-43.

Steel, R.G.D. and J.H. Torrie. 1960. Principles and procedures of statistics. McGraw-Hill, New York.

Strehler, B.L. and W.A. Arnold. 1951. Light production by green plants. J. Gen. Physiol. 34:809-820.

Vapaavuori, E. M., E. Korpilahti, and A.H. Nurmi. 1984. Photosynthetic rate in willow leaves during water stress and changes in the chloroplast ultrastructure with special references to crystal inclusions. J. Expt. Bet. 35:306-321. 